\title{
A Geometric Modeling Method Based on TH-Type Uniform B-Splines
}

\author{
Jin Xie $\mathrm{Xi}^{1,2}$ \\ ${ }^{1}$ Department of Mathematics and Physics, Hefei University, Hefei 230601, China \\ ${ }^{2}$ Department of Mathematics and Physics, University of La Verne, La Verne, CA 91750, USA
}

Correspondence should be addressed to Jin Xie; hfuuxiejin@126.com

Received 26 January 2014; Accepted 24 May 2014; Published 15 June 2014

Academic Editor: Vassilios C. Loukopoulos

Copyright (C) 2014 Jin Xie. This is an open access article distributed under the Creative Commons Attribution License, which permits unrestricted use, distribution, and reproduction in any medium, provided the original work is properly cited.

\begin{abstract}
A geometric modeling method based on TH-type uniform B-splines which are composed of trigonometric and hyperbolic polynomial with parameters is introduced in this paper. The new splines possess many important properties of quadratic and cubic B-splines. Taking different values of the parameters, one can not only locally adjust the shape of the curves, but also change the type of some segments of a curve between trigonometric and hyperbolic functions as well. The given curves can also interpolate directly control polygon locally by selecting special parameters. Moreover, the introduced splines can represent some quadratic curves and transcendental curves with selecting proper control points and parameters.
\end{abstract}

\section{Introduction}

B-splines are used as an important geometric modeling tool in computer aided geometric design (CAGD). However, there are still several limitations on B-splines in practical applications [1]. Firstly, for the fixed control points and the knot sequences, the shape of the curves and surfaces represented by B-splines is fixed. Secondly, the B-splines cannot represent conics (except parabolas) and some known curves such as the cycloid and the helix exactly. Although NURBS can overcome the shortcomings of B-splines, as the complexity of its rational basis functions and its derivatives and integrals are hard to compute, it is not convenient to the user. So, in order to avoid their inconveniences, recently, several new splines defined in different space from the usual polynomial space have been proposed for geometric modeling in CAGD [2-11]. T-type splines were introduced [2-7], which can exactly represent the ellipse, the cycloid, and the helix. Pottmann and Wagner [8] and Koch and Lyche [9] presented a kind of exponential splines in tension in that space $\{1, t, \cosh t, \sinh t\}$. Lü et al. [10] gave the explicit expressions for uniform splines. Li and Wang [11] generalized the curves and surfaces of exponential forms to algebraic hyperbolic spline forms of any degree, which can represent exactly some remarkable curves such as the hyperbola and the catenary. However, $\mathrm{H}$-type uniform Bsplines in tension are not applicable to freeform polynomial curves of high orders, which severely restrict their applications in CAGD.

By comparing T-type uniform B-splines and $\mathrm{H}$-type uniform B-splines, we found that T-type uniform B-splines are located on one side of the $\mathrm{B}$-spline, and $\mathrm{H}$-type uniform $\mathrm{B}$ splines are located on the other side of the B-spline. Therefore, one thinks if the two different curves can be unified to produce new blending splines, then the new curve will have more plentiful modeling power. In order to construct more flexible curves for curves and surface modeling, Zhang et al. $[12,13]$ proposed a curve family, named FB-spline, that uses a unified basis $\{1, t, \cos t, \sin t\}$ and basis $\{1, t, \cosh t, \sinh t\}$. FB-splines inherited nearly all the properties that the T-type $\mathrm{B}$-splines and the $\mathrm{H}$-type $\mathrm{B}$-splines have. However, the formulas for the FB-splines were rather complicated. Wang and Fang [14] unified and extended three types of splines by a new kind of spline (UE-spline for short) defined over the space $\left\{\cos \omega_{i} t, \sin \omega_{i} t, 1, t, \ldots, t^{l}, \ldots\right\}$, where the type of a curve can be switched by a frequency sequence $\left\{\omega_{i}\right\}$. However, the geometric meaning of the sequence $\left\{\omega_{i}\right\}$ is not obvious. Over the space $\operatorname{span}\left\{\sin t, \cos t, \sinh t, \cosh t, 1, t, \ldots, t^{n-5}\right\}, n \geq 5$. $\mathrm{Xu}$ and Wang [15] presented two new unified mathematics 
models of conics and polynomial curves, called algebraic hyperbolic trigonometric (AHT) Bézier curves and nonuniform algebraic hyperbolic trigonometric (NUAHT) B-spline curves of order $n$, which share most of the properties as those of the Bézier curves and B-spline curves in polynomial space.

In this paper, we present a new geometric modeling method based on two kinds of TH-type uniform B-splines which are composed of hyperbolic and trigonometric functions. The introduced spline has the following features: (1) the new spline curves can be adjusted totally or locally. (2) The given curves can switch into T-type B-spline curves or $\mathrm{H}$-type B-spline curves when the parameter is equal to 0 or 1 . (3) Without solving the system of equations, the new curves can interpolate certain control points directly. (4) The THtype B-spline curves can be used to represent some conics and transcendental curves with the parameters and control points chosen properly.

The rest of this paper is organized as follows. In Sections 2 and 3 , the TH-type basis functions and corresponding THtype curves are established and the properties of the basis functions are proved. In Section 4, some properties of the TH-type B-spline curves are discussed. It is pointed out in Section 5 that some transcendental curves can be represented precisely with the TH-type curves, and the applications of the curves are shown in Section 6.

\section{Quadratic TH-Type B-Spline}

Definition 1. Given $t \in[0,1]$, the quadratic basis functions based on weighted trigonometric and hyperbolic polynomials are as follows:

$$
\begin{aligned}
\mathrm{qth}_{0,2}\left(t ; \lambda_{i}\right)= & \frac{1}{2}-\lambda_{i} \frac{2 e \cosh (1-t)-e^{2}-1}{2(e-1)^{2}} \\
& +\frac{1}{2}\left(\lambda_{i}-1\right) \sin \frac{\pi t}{2}, \\
\mathrm{qth}_{1,2}\left(t ; \lambda_{i}, \lambda_{i+1}\right)= & \frac{1}{2}\left(1-\lambda_{i}\right) \sin \frac{\pi t}{2} \\
& +\lambda_{i} \frac{2 e(\cosh 1-\cosh (1-t))}{(e-1)^{2}} \\
& +\lambda_{i+1} \frac{2 e(\cosh 1-\cosh (t))}{(e-1)^{2}} \\
& +\frac{1}{2}\left(1-\lambda_{i+1}\right) \cos \frac{\pi t}{2}, \\
\mathrm{qth}_{2,2}\left(t ; \lambda_{i+1}\right)= & \lambda_{i+1} \frac{e(\cosh (t)-1)}{2(e-1)^{2}} \\
& +\frac{1}{2}\left(1-\lambda_{i+1}\right)\left(1-\cos \frac{\pi t}{2}\right),
\end{aligned}
$$

which are named the basis functions of quadratic TH-type B-spline.

Theorem 2. The above functions have the following properties.

(i) Partition of unity: $q t h_{0,2}\left(t ; \lambda_{i}\right)+q t h_{1,2}\left(t ; \lambda_{i}, \lambda_{i+1}\right)+$ $q t h_{2,2}\left(t ; \lambda_{i+1}\right)=1$. (ii) Symmetry: $q t h_{0,2}\left(t ; \lambda_{i}\right)=q t h_{2,2}\left(1-t ; \lambda_{i}\right), q t h_{1,2}\left(t ; \lambda_{i}\right.$, $\left.\lambda_{i+1}\right)=q t h_{1,2}\left(1-t ; \lambda_{i+1}, \lambda_{i}\right)$.

(iii) Nonnegativity: if $(1+\sqrt{e})^{2} /\left((1-\sqrt{e})(1+\sqrt{e})^{2}+\sqrt{2 e}\right) \leq$ $\lambda_{i}, \lambda_{i+1} \leq(e-1)^{2} \pi^{2} /\left((e-1)^{2} \pi^{2}-8 e\right)$, then $q t_{k, 2}(t) \geq$ $0, k=0,1,2$.

Proof. (i) and (ii) are easy to be proved by simple computation. Next we will prove (iii).

By direct computation, we have $\mathrm{qth}_{0,2}\left(0 ; \lambda_{i}\right)=$ $1, \mathrm{qth}_{0,2}\left(1 ; \lambda_{i}\right)=0$. And since $0 \leq t \leq 1, \lambda_{i} \leq$ $(e-1)^{2} \pi^{2} /\left((e-1)^{2} \pi^{2}-8 e\right)$ and $\mathrm{qth}_{0,2}^{\prime}\left(t ; \lambda_{i}\right) \leq 0$, then we have $\mathrm{qth}_{0,2}\left(t ; \lambda_{i}\right) \geq 0$. Evidenced by the same token, we have $\mathrm{qth}_{2,2}\left(t ; \lambda_{i+1}\right) \geq 0$.

From (ii), we have $\mathrm{qth}_{1,2}\left(t ; \lambda_{i}, \lambda_{i+1}\right)=1-\mathrm{qth}_{0,2}\left(t ; \lambda_{i}\right)-$ $\mathrm{qth}_{2,2}\left(t ; \lambda_{i+1}\right)$. Obviously, if we can prove $\mathrm{qth}_{0,2}\left(t ; \lambda_{i}\right)+$ $\mathrm{qth}_{2,2}\left(t ; \lambda_{i+1}\right) \leq 1$, we can prove $\mathrm{qth}_{1,2}\left(t ; \lambda_{i+1}\right) \geq 0$.

Let $f\left(t ; \lambda_{i}, \lambda_{i+1}\right)=\mathrm{qth}_{0,2}\left(t ; \lambda_{i}\right)+\mathrm{qth}_{2,2}\left(t ; \lambda_{i+1}\right)$; we have $f\left(0 ; \lambda_{i}, \lambda_{i+1}\right)=f\left(1 ; \lambda_{i}, \lambda_{i+1}\right)=1$. Thus, when $\lambda_{i}, \lambda_{i+1} \geq(1+$ $\sqrt{e})^{2} /\left((1-\sqrt{e})(1+\sqrt{e})^{2}+\sqrt{2 e}\right)$, we can get

$$
f^{\prime}\left(t ; \lambda_{i}, \lambda_{i+1}\right)= \begin{cases}<0, & t \in[0,0.5), \\ =0, & t=0.5, \\ >0, & t \in(0.5,1] .\end{cases}
$$

So the maximum value of the function $f\left(t ; \lambda_{i}, \lambda_{i+1}\right)$ equals 1. That is, $\mathrm{qth}_{0,2}\left(t ; \lambda_{i}\right)+\mathrm{qth}_{2,2}\left(t ; \lambda_{i+1}\right) \leq 1$, which means $\mathrm{qth}_{1,2}\left(t ; \lambda_{i+1}\right) \geq 0$.

Definition 3. Given control points $P_{i} \in R^{d}(d=2,3, i=$ $0,1, \ldots, n)$, the curves

$$
\begin{aligned}
& \mathrm{QTH}_{i, 2}\left(t ; \lambda_{i}, \lambda_{i+1}\right) \\
& =P_{i-1} \mathrm{qth}_{0,2}\left(t ; \lambda_{i}\right) \\
& +P_{i} \mathrm{qth}_{1,2}\left(t ; \lambda_{i}, \lambda_{i+1}\right)+P_{i+1} \mathrm{qth}_{2,2}\left(t ; \lambda_{i+1}\right), \\
& \quad t \in[0,1], \quad i=1,2, \ldots, n-1
\end{aligned}
$$

are defined quadratic TH-type B-spline curve segments with shape parameters $\lambda_{i}$ and $\lambda_{i+1}$, where $\mathrm{qth}_{0,2}\left(t ; \lambda_{i}\right)$, $\mathrm{qth}_{1,2}\left(t ; \lambda_{i}, \lambda_{i+1}\right)$, and $\mathrm{qth}_{2,2}\left(t ; \lambda_{i+1}\right)$ are the bases of quadratic TH-type B-spline.

\section{Cubic TH-Type B-Spline}

By a similar method, we may define the bases of cubic THtype B-spline.

Definition 4. For $(e-1)^{2} /\left((e-1)^{2}-\pi\right) \leq \lambda_{i}, \leq \lambda_{i+1} \leq(e-$ $1)^{2} \pi^{2} /\left((e-1)^{2} \pi^{2}-8 e\right)$ and $t \in[0,1]$, the following functions,

$$
\begin{aligned}
\operatorname{cth}_{0,3}\left(t ; \lambda_{i}\right)= & \frac{1}{\pi}\left(\lambda_{i}-1\right) \cos \frac{\pi}{2} t+\frac{1}{(e-1)^{2}} \\
& \times\left(\left(2(e-1)^{2}-\left(1+e^{2}\right) \lambda_{i}\right)\right. \\
& \left.\quad \times(1-t)-2 e \lambda_{i} \sinh (1-t)\right),
\end{aligned}
$$




$$
\begin{aligned}
& \operatorname{cth}_{1,3}\left(t ; \lambda_{i}, \lambda_{i+1}\right)=\frac{(e-1)^{2}-\left(1+e^{2}\right) \lambda_{i+1}}{2(e-1)^{2}} \\
& -\frac{(e-1)^{2}-\left(1+e^{2}\right)\left(2 \lambda_{i}+\lambda_{i+1}\right)}{2(e-1)^{2}} \\
& \times(1-t)+\frac{2}{\pi}\left(1-\lambda_{i}\right) \cos \frac{\pi t}{2} \\
& -\frac{1}{\pi}\left(1-\lambda_{i+1}\right) \sin \frac{\pi t}{2} \\
& +\frac{(e+1) \lambda_{i+1}}{2(e-1)} \cosh (1-t) \\
& -\frac{\left(1+e^{2}\right) \lambda_{i+1}+4 e \lambda_{i}}{(e-1)^{2} \pi} \sinh (1-t), \\
& \operatorname{cth}_{2,3}\left(t ; \lambda_{i}, \lambda_{i+1}\right)=\frac{(e-1)^{2}-\left(1+e^{2}\right) \lambda_{i}}{2(e-1)^{2}} \\
& -\frac{(e-1)^{2}-\left(1+e^{2}\right)\left(\lambda_{i}+2 \lambda_{i+1}\right)}{2(e-1)^{2}} t \\
& +\frac{2}{\pi}\left(1-\lambda_{i+1}\right) \sin \frac{\pi t}{2} \\
& -\frac{1}{\pi}\left(1-\lambda_{i}\right) \cos \frac{\pi t}{2}+\frac{(e+1) \lambda_{i}}{2(e-1)} \cosh t \\
& -\frac{\left(1+e^{2}\right) \lambda_{i}+4 e \lambda_{i+1}}{(e-1)^{2}} \sinh t \\
& \operatorname{cth}_{3,3}\left(t ; \lambda_{i+1}\right)=\frac{1}{\pi}\left(\lambda_{i+1}-1\right) \sin \frac{\pi t}{2}+\frac{1}{2(e-1)^{2}} \\
& \times\left(\left((e-1)^{2}-\left(1+e^{2}\right) \lambda_{i+1}\right) t\right. \\
& \left.+2 e \lambda_{i+1} \sinh t\right) \text {, }
\end{aligned}
$$

are called basis functions of cubic TH-type B-spline with shape parameters $\lambda_{i}$ and $\lambda_{i+1}$.

It is easy to prove that the basis functions of cubic TH-type B-spline have the same properties: nonnegativity, partition of unity, and symmetry.

Definition 5. Given control points $P_{i} \in R^{d}(d=2,3, i=$ $0,1, \ldots, n)$, the curves

$$
\begin{aligned}
& \mathrm{CTH}_{i, 3}\left(t ; \lambda_{i}, \lambda_{i+1}\right) \\
& =P_{i-1} \operatorname{cth}_{0,3}\left(t ; \lambda_{i}\right)+P_{i} \operatorname{cth}_{1,3}\left(t ; \lambda_{i}, \lambda_{i+1}\right) \\
& +P_{i+1} \operatorname{cth}_{2,3}\left(t ; \lambda_{i}, \lambda_{i+1}\right)+P_{i+2} \operatorname{cth}_{3,3}\left(t ; \lambda_{i+1}\right), \\
& \quad t \in[0,1], \quad i=1,2, \ldots, n-1
\end{aligned}
$$

are defined cubic TH-type B-spline curve segments with shape parameters $\lambda_{i}$ and $\lambda_{i+1}$, where $\operatorname{cth}_{0,3}\left(t ; \lambda_{i}\right)$, $\operatorname{cth}_{1,3}\left(t ; \lambda_{i}, \lambda_{i+1}\right), \operatorname{cth}_{2,3}\left(t ; \lambda_{i}, \lambda_{i+1}\right)$, and $\mathrm{cth}_{3,3}\left(t ; \lambda_{i+1}\right)$ are the basis functions of cubic TH-type B-spline.

\section{The Properties of the TH-Type B-Spline Curves}

According to the properties of the basis functions and definition, it is easy to get the following properties of curves (3) and (5).

(i) Continuity

Theorem 6. For the uniform knots, the curves (3) are $C^{1}$ continuous and the curves (5) are $C^{2}$ continuous.

Proof. For the curve (3), we can get

$$
\begin{aligned}
& \mathrm{QTH}_{i, 2}\left(0 ; \lambda_{i}, \lambda_{i+1}\right)=\frac{1}{2}\left(P_{i-1}+P_{i}\right), \\
& \mathrm{QTH}_{i, 2}\left(1 ; \lambda_{i}, \lambda_{i+1}\right)=\frac{1}{2}\left(P_{i}+P_{i+1}\right), \\
& \mathrm{QTH}_{i, 2}^{\prime}\left(0 ; \lambda_{i}, \lambda_{i+1}\right) \\
& =\frac{(1-e) \pi+(e(\pi-2)-2-\pi) \lambda_{i}}{4(e-1)}\left(P_{i}-P_{i-1}\right), \\
& \mathrm{QTH}_{i, 2}^{\prime}\left(1 ; \lambda_{i}, \lambda_{i+1}\right) \\
& =\frac{(1-e) \pi+(e(\pi-2)-2-\pi) \lambda_{i+1}}{4(e-1)}\left(P_{i+1}-P_{i}\right) .
\end{aligned}
$$

Thus, we obtain $\mathrm{QTH}_{i-1,2}^{(k)}\left(1 ; \lambda_{i}, \lambda_{i+1}\right)=\mathrm{QTH}_{i, 2}^{(k)}\left(0 ; \lambda_{i}, \lambda_{i+1}\right)$ $(k=0,1)$; that is to say, the curves $(3)$ are $C^{1}$ continuous.

For the curves (5), we get

$$
\begin{aligned}
& \mathrm{CTH}_{i, 3}\left(0 ; \lambda_{i}, \lambda_{i+1}\right) \frac{(e-1)^{2}\left(\pi+2 \lambda_{i}-2\right)-2 \pi \lambda_{i}}{2(e-1)^{2} \pi}\left(P_{i-1}+P_{i+1}\right) \\
&= \\
&+\frac{2(e-1)^{2}\left(\pi-\lambda_{i}-2\right)+2 \pi \lambda_{i}}{(e-1)^{2} \pi} P_{i}, \\
& \mathrm{CTH}_{i, 3}\left(1 ; \lambda_{i}, \lambda_{i+1}\right) \frac{(e-1)^{2}\left(\pi+2 \lambda_{i+1}-2\right)-2 \pi \lambda_{i+1}}{2(e-1)^{2} \pi}\left(P_{i}+P_{i+2}\right) \\
&=+\frac{2(e-1)^{2}\left(\pi-\lambda_{i+1}-2\right)+2 \pi \lambda_{i+1}}{(e-1)^{2} \pi} P_{i+1}, \\
& \quad \\
& \mathrm{CTH}_{i, 3}^{\prime}\left(0 ; \lambda_{i}, \lambda_{i+1}\right)=\frac{1}{2}\left(P_{i+1}-P_{i-1}\right),
\end{aligned}
$$




$$
\begin{aligned}
& \mathrm{CTH}_{i, 3}^{\prime}\left(1 ; \lambda_{i}, \lambda_{i+1}\right)=\frac{1}{2}\left(P_{i+2}-P_{i}\right), \\
& \mathrm{CTH}_{i, 3}^{\prime \prime}\left(0 ; \lambda_{i}, \lambda_{i+1}\right) \\
& =\frac{(e-1) \pi+((e-1) \pi-2(e+1)) \lambda_{i}}{4(e-1)} \\
& \quad \times\left(P_{i-1}-2 P_{i}+P_{i+1}\right), \\
& \mathrm{CTH}_{i, 3}^{\prime \prime}\left(1 ; \lambda_{i}, \lambda_{i+1}\right) \\
& =\frac{(e-1) \pi+((e-1) \pi-2(e+1)) \lambda_{i+1}}{4(e-1)} \\
& \quad \times\left(P_{i}-2 P_{i+1}+P_{i+2}\right) .
\end{aligned}
$$

So, we have $\mathrm{CTH}_{i-1,3}^{(k)}\left(1 ; \lambda_{i}, \lambda_{i+1}\right)=\mathrm{CTH}_{i, 3}^{(k)}\left(0 ; \lambda_{i}, \lambda_{i+1}\right)(k=$ $0,1,2)$. This implies that curves $(5)$ are $C^{2}$ continuous.

This implies the theorem.

(ii) Local Adjustable Properties. From formulas (3) and (5), the parameter $\lambda_{i}$ only affects two curve segments without altering the remainder. Figure 1 shows local adjustable quadratic uniform TH-type spline curves, where all parameters $\lambda_{i}=$ 0.5 in the solid curves and all parameters $\lambda_{i}=0.5$ except $\lambda_{3}=-1$ in the dotted curves. The parameter only affects the 2 th and the 3 th curve segment. Figure 2 shows the local adjustable cubic uniform TH-type spline curves, where all the parameters are equal to 0.5 in the solid curves, and all the parameters are equal to 0.5 except $\lambda_{5}=-1$ in the dotted curves. The parameter $\lambda_{5}$ only affects the 4 th and 5 th curve segment.

Obviously, when all parameters $\lambda_{i}$ are the same, the curves can be adjusted totally.

(iii) Local Interpolating Properties. For the curve (3), letting $\lambda_{i}=\lambda_{i+1}=(\sqrt{e}+1)^{2} /(1-\sqrt{2 e}+e) \doteq 5.05952$, then $\mathrm{qth}_{i, 2}(0.5)=P_{i}$; that is, the curve interpolates the point $P_{i}$. For the curve (5), when $\lambda_{i}=(2-\pi)(e-1)^{2} /(2(e-$ $\left.1)^{2}-2 \pi\right) \doteq 8.91206, \operatorname{cth}_{i, 3}(0)=P_{i}, \lambda_{i+1}=(2-\pi)(e-$ $1)^{2} /\left(2(e-1)^{2}-2 \pi\right) \doteq 8.91206, \operatorname{cth}_{i, 3}(1)=P_{i+1}$; that is, the curve interpolates the points $P_{i}$ and $P_{i+1}$. Figure 3 shows local interpolating quadratic TH-type spline curves, where the curve interpolates the point $P_{5}$ when the parameter $\lambda_{5}=\lambda_{6}=$ 5.05952. The local interpolating cubic TH-type spline curves are showed in Figure 4, where the curves interpolate the point $P_{5}$ when the parameter $\lambda_{5}=8.91206$.

\section{The Representations of Some Known Curves}

When the parameters $\lambda_{i}=\lambda_{i+1}=0$, the curves (3) and (5) are T-type uniform B-spline curves. If the parameters $\lambda_{i}=\lambda_{i+1}=$ 1 , the curves (3) and (5) become H-type uniform B-splines.

5.1. The Representation of the Conic Curves. The ellipse and hyperbola are the most common in the conic curve. If the

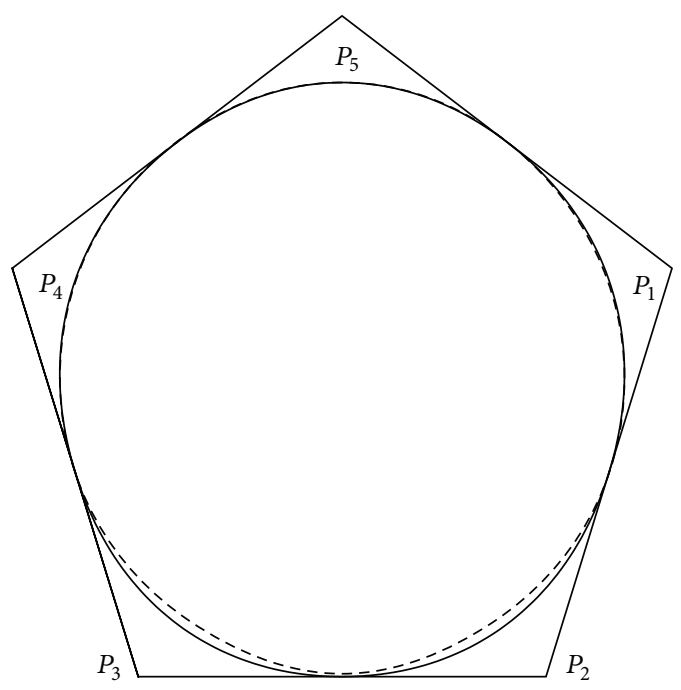

FIGURE 1: Local adjustable quadratic uniform TH-type spline curves.

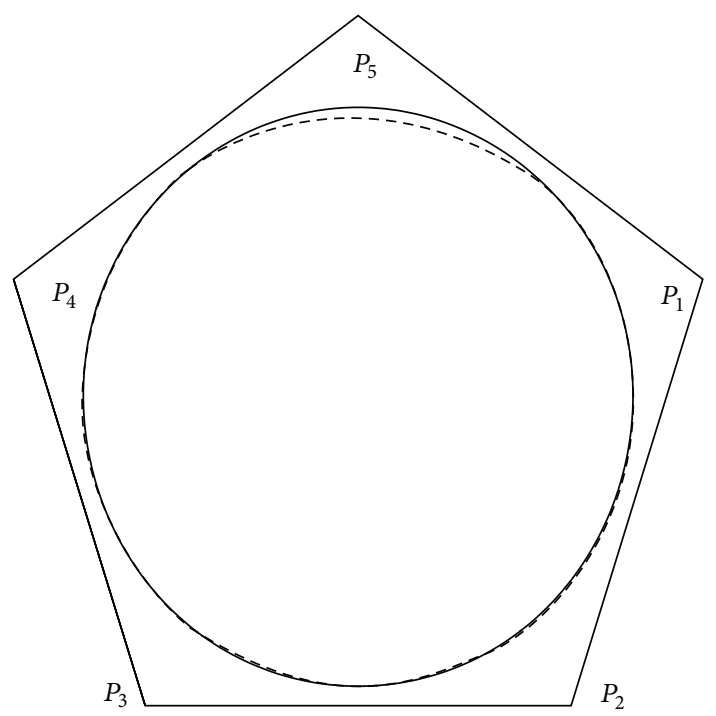

FIGURE 2: Local adjustable cubic uniform TH-type spline curves.

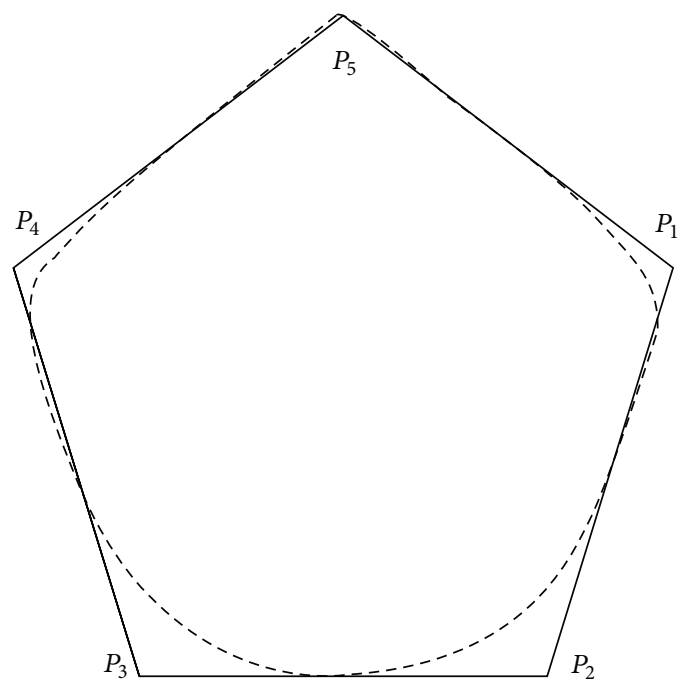

FIgURE 3: Local interpolating quadratic TH-type spline curves. 


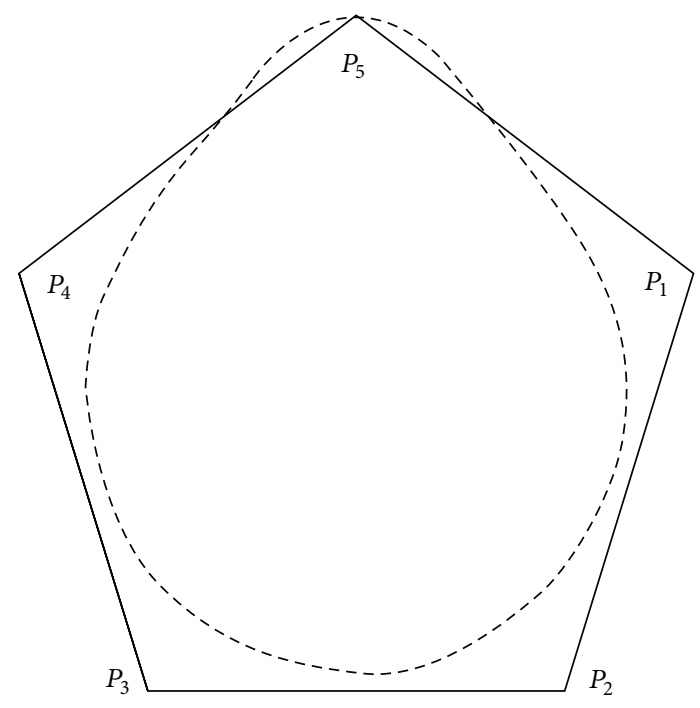

FIGURE 4: Local interpolating cubic uniform TH-type spline curves.

control points and the parameters are selected properly, the curves (3) and (5) can represent them precisely.

Given the uniform knots, for the quadratic T-type Bspline curve, we take the coordinates of the points $P_{i-1}, P_{i}$, and $P_{i+1}$ as follows:

$$
\begin{aligned}
P_{i-1} & =(m-a, n+b), \\
P_{i} & =(m+a, n+b), \\
P_{i+1} & =(m+a, n-b),
\end{aligned}
$$

$$
(a b \neq 0) \text {. }
$$

For the cubic T-type B-spline curve, we take

$$
\begin{aligned}
& P_{i-1}=\left(m, n-\frac{\pi}{2} b\right), \\
& P_{i}=\left(m+\frac{\pi}{2} a, n\right), \\
& P_{i+1}=\left(m, n-\frac{\pi}{2} b\right), \\
& P_{i+2}=\left(m-\frac{\pi}{2} a, n\right), \\
&(a b \neq 0) .
\end{aligned}
$$

Then, when $t \in[0,1]$ and $\lambda_{i}=\lambda_{i+1}=0$, we obtain a parametric equation as follows:

$$
\begin{aligned}
& x(t)=m+a \cos \frac{\pi}{2} t, \\
& y(t)=n+b \sin \frac{\pi}{2} t .
\end{aligned}
$$

It is the parametric form of the ellipse; see Figure 5. In order to represent the hyperbola, for the quadratic $\mathrm{H}$-type uniform $\mathrm{B}$-spline curves, the control points are taken as follows:

$$
\begin{aligned}
& P_{i-1}=\left(m+a, n+\frac{1-e}{1+e} b\right), \\
& P_{i}=\left(m+a, n \frac{1-e}{1+e} b\right), \\
& P_{i+1}=\left(m+\frac{e^{2}-e+1}{e} a, n+\frac{e^{3}-1}{e^{2}+e} b\right), \\
&(a b \neq 0) .
\end{aligned}
$$

For the cubic H-type uniform B-spline curves, we take $P_{i-1}=\left(m+\left(\left(e^{2}+1\right) / e\right) a, n-\left(\left(e^{4}+1\right) /\left(e^{3}-e\right)\right) b\right), P_{i}=(m+$ $\left.a, n+\left(\left(e^{2}+1\right) /\left(e^{2}-1\right)\right) b\right), P_{i+1}=\left(m, n+\left(2 e /\left(e^{2}-1\right)\right) b\right)$, $P_{i+2}=\left(m-a,\left(\left(e^{2}+1\right) /\left(e^{2}-e\right)\right) b\right)(a b \neq 0)$ as control points. So we get a parametric equation as follows:

$$
\begin{aligned}
& x(t)=m+a \cosh t, \\
& y(t)=n+b \sinh t,
\end{aligned}
$$

which represents an arc of the hyperbola; see Figure 6.

5.2. The Representation of the Transcendental Curves. In this section, we can represent the transcendental curves with the uniform TH-type B-splines, such as cycloid and catenary.

When parameters $\lambda_{i}=\lambda_{i+1}=0$, control points are taken as follows:

$$
\begin{aligned}
& P_{i-1}=\left(\frac{\pi-2}{4} a, \frac{4+\pi}{4} a\right), \\
& P_{i}=\left(\frac{2-\pi}{4} a, \frac{4-\pi}{4} a\right), \\
& P_{i+1}=\left(\frac{6-3 \pi}{4} a, \frac{\pi+4}{4} a\right), \\
& P_{i+2}=\left(\frac{10-\pi}{4} a, \frac{4+3 \pi}{4} a\right), \\
&(a \neq 0) .
\end{aligned}
$$

So we obtain the parametric equation as follows:

$$
\begin{aligned}
& x(t)=a\left(t-\sin \frac{\pi}{2} t\right), \\
& y(t)=a\left(1-\cos \frac{\pi}{2} t\right),
\end{aligned}
$$

which represents an arc of a cycloid; see Figure 7.

Similarly, when taking $P_{i-1}=\left(m+2 a, n+\left(\left(e^{4}+1\right) /\left(e^{3}-\right.\right.\right.$ e) $) b), P_{i}=\left(m+a, n+\left(\left(e^{2}+1\right) /\left(e^{2}-1\right)\right)\right), P_{i+1}=\left(m, n+\left(2 e /\left(e^{2}-\right.\right.\right.$ $1)) b)$, and $P_{i+2}=\left(m-a, n+\left(\left(e^{2}+1\right) /\left(e^{2}-1\right)\right) b\right)(a b \neq 0)$ as control points, the parameters $\lambda_{i}=\lambda_{i+1}=1$. By formula (5), we have the following equation:

$$
\begin{gathered}
x(t)=m+a t, \\
y(t)=n+b \cosh t,
\end{gathered}
$$

which is the parametric equation of the catenary; see Figure 8. 


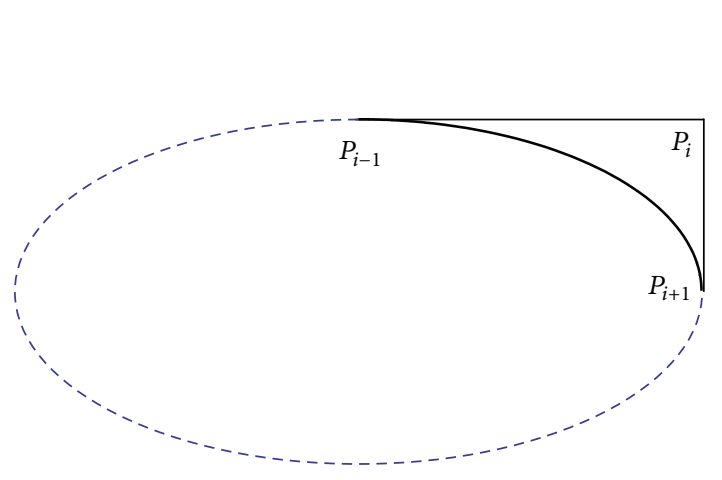

(a)

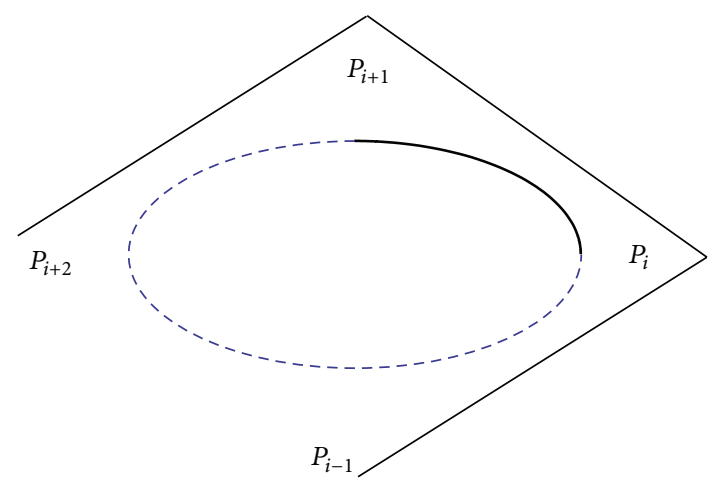

(b)

FIGURE 5: The representation of ellipse with quadratic (a) and cubic (b) T-type B-spline curves.

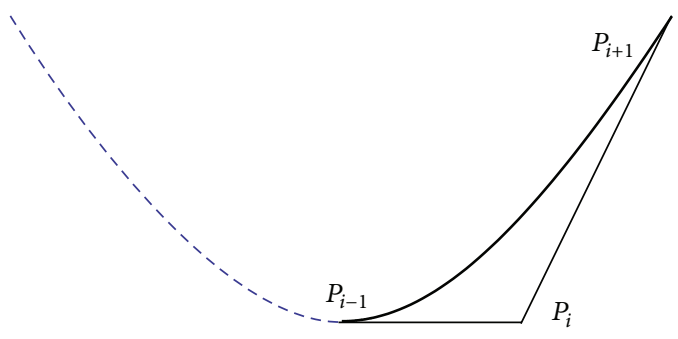

(a)

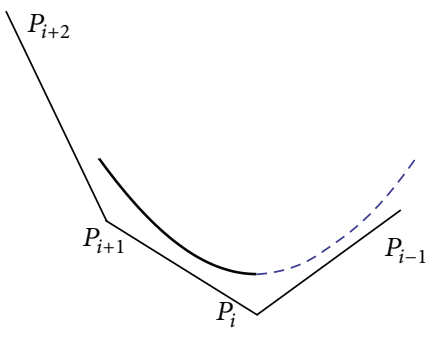

(b)

FIgURE 6: The representation of hyperbola with quadratic (a) and cubic (b) H-type B-spline curves.

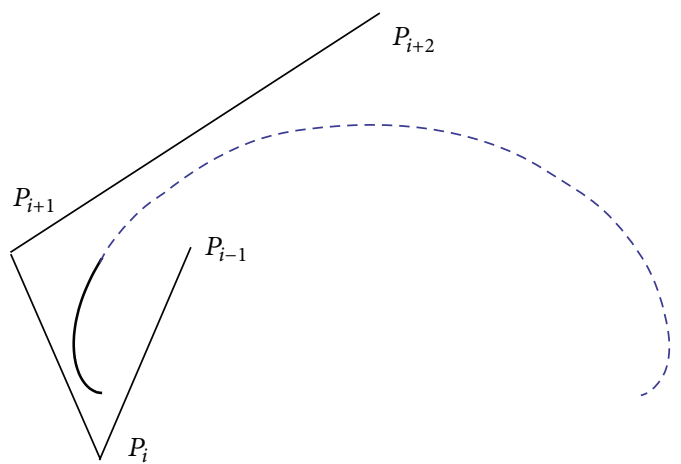

Figure 7: The representation of cycloid with cubic T-type B-spline curves.

\section{The Applications of the TH-Type Splines}

From the last section, we see, letting the parameter be equal to 0 or 1 , the types of the curves can be switched easily. So, by selecting control points and parameters properly, we can represent different type curve segments among a blending curve. In Figure 9, a closed $C^{1}$ blending curve is composed of different type curves with the quadratic TH-type B-splines, where the coordinates of control points are $P_{0}=P_{6}=(-3,(e-$ $1) /(e+1)), P_{1}=P_{7}=(-3,(1-e) /(e+1)), P_{2}=(3,(e-1) /(e+$ $1)), P_{3}=(3,(1-e) /(e+1)), P_{4}=\left(4-e-(1 / e),\left(e^{3}-1\right) /\left(e^{2}+e\right)\right)$, $P_{5}=\left(e-4+(1 / e),\left(e^{3}-1\right) /\left(e^{2}+e\right)\right)$ and the parameters

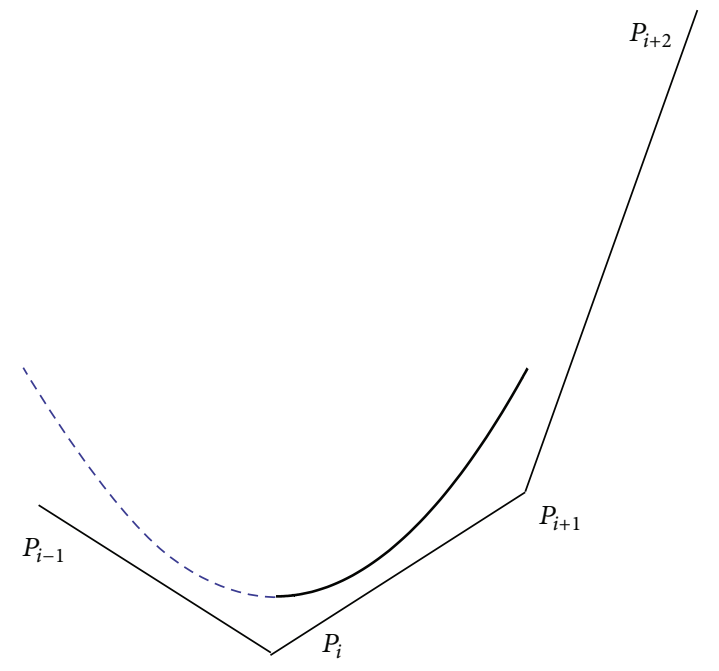

FIgURE 8: The representation of catenary with cubic H-type B-spline curves.

$\lambda_{i}=(0,0,1,1,5.05952,5.05952,0)(i=1,2, \ldots, 7)$. The 1 st segment is a trigonometric curve, which is a quarter of a parabola. The 3rd segment is a hyperbola arc. The blending curve interpolates the point $P_{5}$ in that the parameters $\lambda_{5}=$ $\lambda_{6}=5.05952$.

Figure 10 shows an open $C^{2}$ blending curve represented by the cubic TH-type B-splines. The control points are taken 


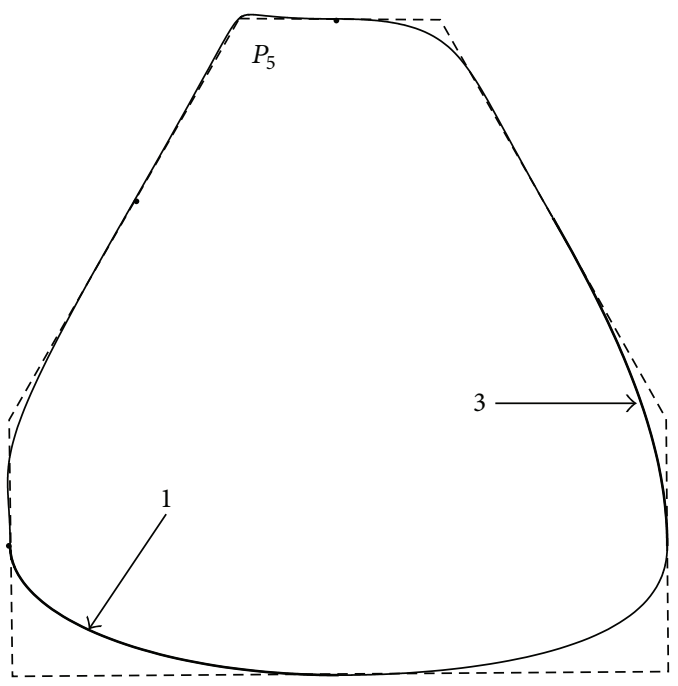

Figure 9: A closed $C^{1}$ blending curve with quadratic TH-type Bsplines.

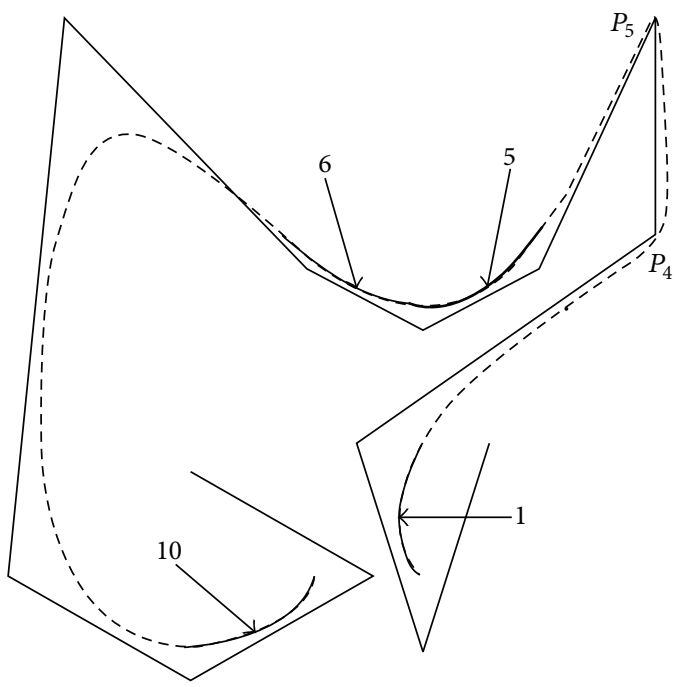

Figure 10: An open $C^{2}$ blending curve with cubic TH-type Bsplines.

as follows: $P_{0}=((\pi-2) / 2,1), P_{1}=(0,(2-\pi) / 2), P_{2}=((2-$ $\pi) / 2,1), P_{3}=(2,(2+\pi) / 2), P_{4}=\left(2,\left(e^{4}+e^{3}-e+1\right) /\left(e^{3}-e\right)\right)$, $P_{5}=\left(1,2 e^{2} /\left(e^{2}-1\right)\right), P_{6}=\left(0,\left(e^{2}+2 e-1\right) /\left(e^{2}-1\right)\right)$, $P_{7}=\left(-1,2 e^{2} /\left(e^{2}-1\right)\right), P_{8}=\left(-\left(e^{2}+1\right) / e,\left(e^{4}+e^{3}-\right.\right.$ $\left.e+1) /\left(e^{3}-e\right)\right), P_{9}=(-(\pi+4) / 2,0), P_{10}=(-2,-\pi / 4)$, $P_{11}=((\pi-4) / 2,0), P_{12}=(-2, \pi / 4)$, where the parameters $\lambda_{i}=(0,0,8.91206,8.91206,1,1,1,1,0.5,0)(i=1,2, \ldots, 11)$. The 1st segment of the bending curve is a trigonometric curve, which is a part of the cycloid. The 5 th and 6 th segment are the catenary and hyperbola, respectively. The 10th segment is the parabola arc. Since the parameters $\lambda_{3}=\lambda_{4}=12.0938$, the blending curve interpolates the points $P_{4}$ and $P_{5}$.

\section{Conflict of Interests}

The author declares that there is no conflict of interests regarding the publication of this paper.

\section{Acknowledgments}

This work was funded by the Natural Science Foundation of Anhui Province of China under Grant no. 1208085MA15, the Key Project Foundation of Scientific Research, Education Department of Anhui Province under Grant no. KJ2014ZD30, and Key Construction Disciplines Foundation of Hefei University under Grant no. 2014XK08.

\section{References}

[1] L. Piegle and W. Tiller, The NURBS Book, Springer, Berlin, Germany, 1995.

[2] J. Zhang, "Two different forms of C-B-splines," Computer Aided Geometric Design, vol. 14, no. 1, pp. 31-41, 1997.

[3] J. Zhang, "C-curves: an extension of cubic curves," Computer Aided Geometric Design, vol. 13, no. 3, pp. 199-217, 1996.

[4] H. Wu and X. Chen, "Cubic non-uniform trigonometric polynomial curves with multiple shape parameters," Journal of Computer-Aided Design and Computer Graphics, vol. 18, no. 10, pp. 1599-1606, 2006 (Chinese).

[5] X. Han, "Cubic trigonometric polynomial curves with a shape parameter," Computer Aided Geometric Design, vol. 21, no. 6, pp. 535-548, 2004.

[6] G. Xu, G. Wang, and W. Chen, "Geometric construction of energy-minimizing Béezier curves," Science China Information Sciences, vol. 54, no. 7, pp. 1395-1406, 2011.

[7] W.-T. Wang and G.-Z. Wang, "Trigonometric polynomial uniform B-spline with shape parameter," Chinese Journal of Computers, vol. 28, no. 7, pp. 1192-1198, 2005 (Chinese).

[8] H. Pottmann and M. G. Wagner, "Helix splines as an example of affine Tchebycheffian splines," Advances in Computational Mathematics, vol. 2, no. 1, pp. 123-142, 1994.

[9] P. E. Koch and T. Lyche, "Exponential B-splines in tension," in Approximation Theory VI, C. K. Chui, L. L. Schumaker, and J. D. Ward, Eds., pp. 361-364, Academic Press, New York, NY, USA, 1989.

[10] Y. Lü, G. Wang, and X. Yang, "Uniform hyperbolic polynomial B-spline curves," Computer Aided Geometric Design, vol. 19, no. 6, pp. 379-393, 2002.

[11] Y.-J. Li and G.-Z. Wang, "Two kinds of B-basis of the algebraic hyperbolic space," Journal of Zhejiang University Science A, vol. 6, no. 7, pp. 750-759, 2005.

[12] J. Zhang, F.-L. Krause, and H. Zhang, "Unifying C-curves and H-curves by extending the calculation to complex numbers," Computer Aided Geometric Design, vol. 22, no. 9, pp. 865-883, 2005.

[13] J. Zhang and F.-L. Krause, "Extending cubic uniform B-splines by unified trigonometric and hyperbolic basis," Graphical Models, vol. 67, no. 2, pp. 100-119, 2005.

[14] G. Wang and M. Fang, "Unified and extended form of three types of splines," Journal of Computational and Applied Mathematics, vol. 216, no. 2, pp. 498-508, 2008.

[15] G. Xu and G.-Z. Wang, "AHT Bézier curves and NUAHT BSpline curves," Journal of Computer Science and Technology, vol. 22, no. 4, pp. 597-607, 2007. 


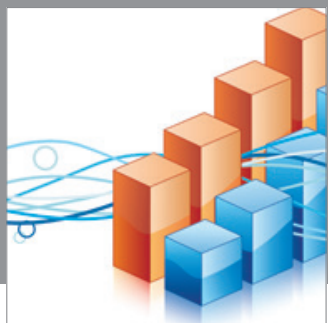

Advances in

Operations Research

mansans

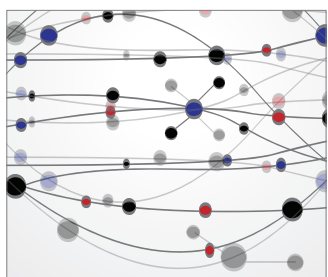

The Scientific World Journal
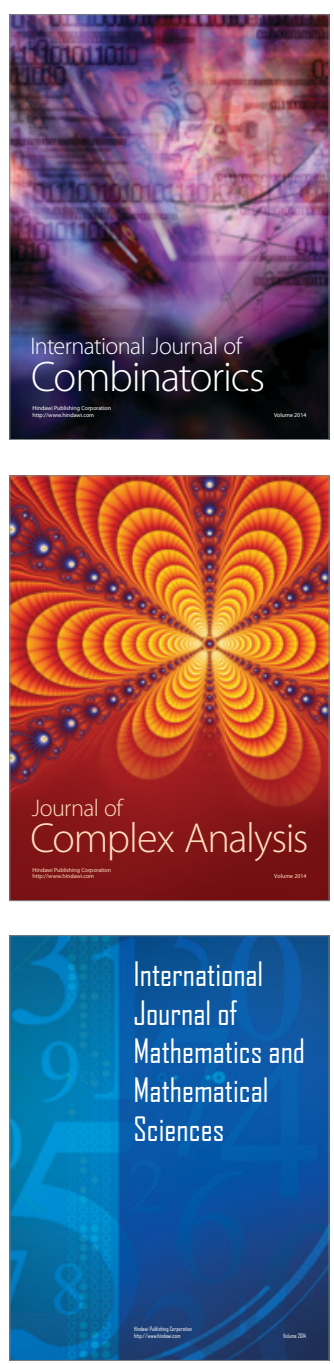
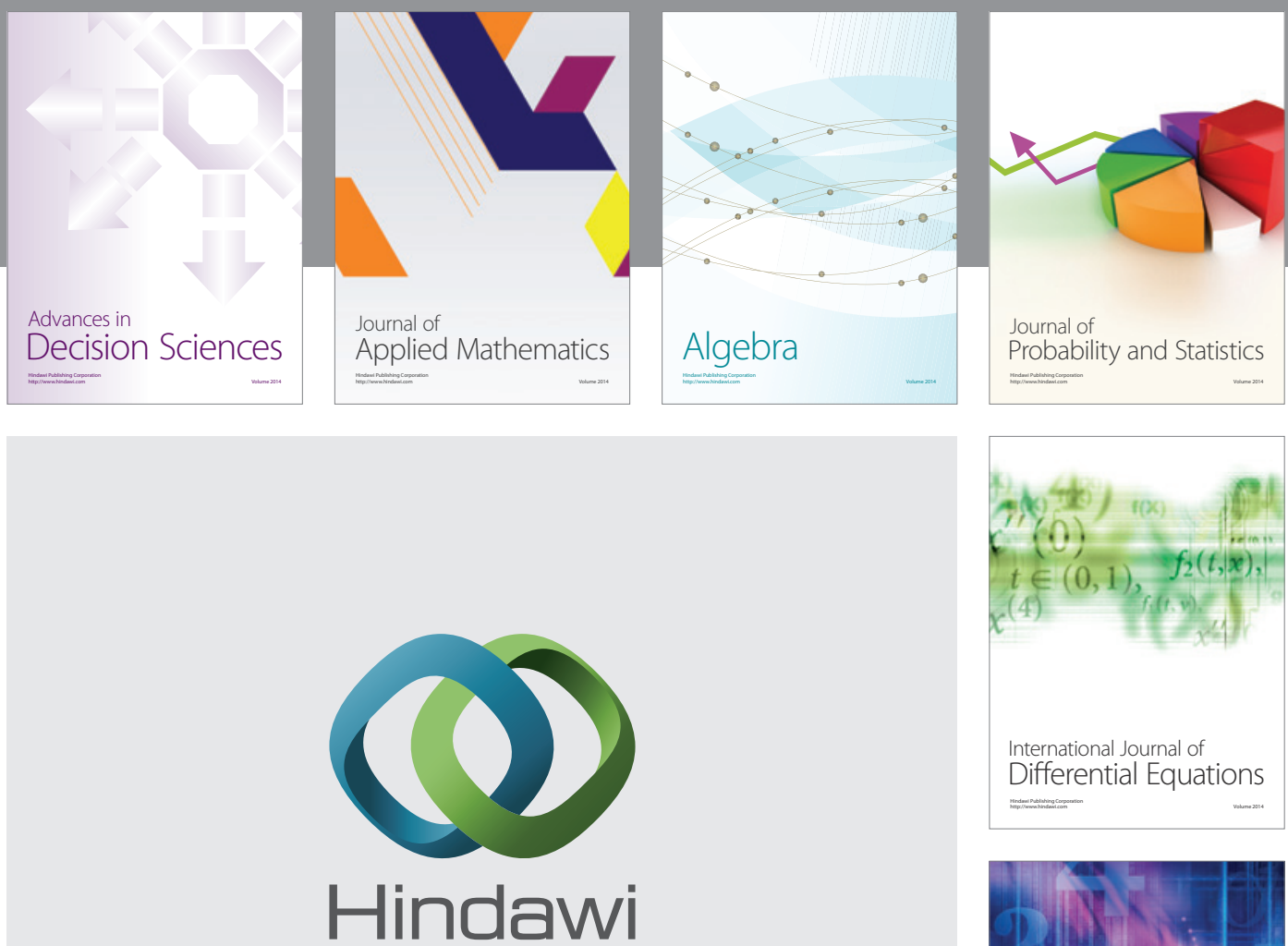

Submit your manuscripts at http://www.hindawi.com
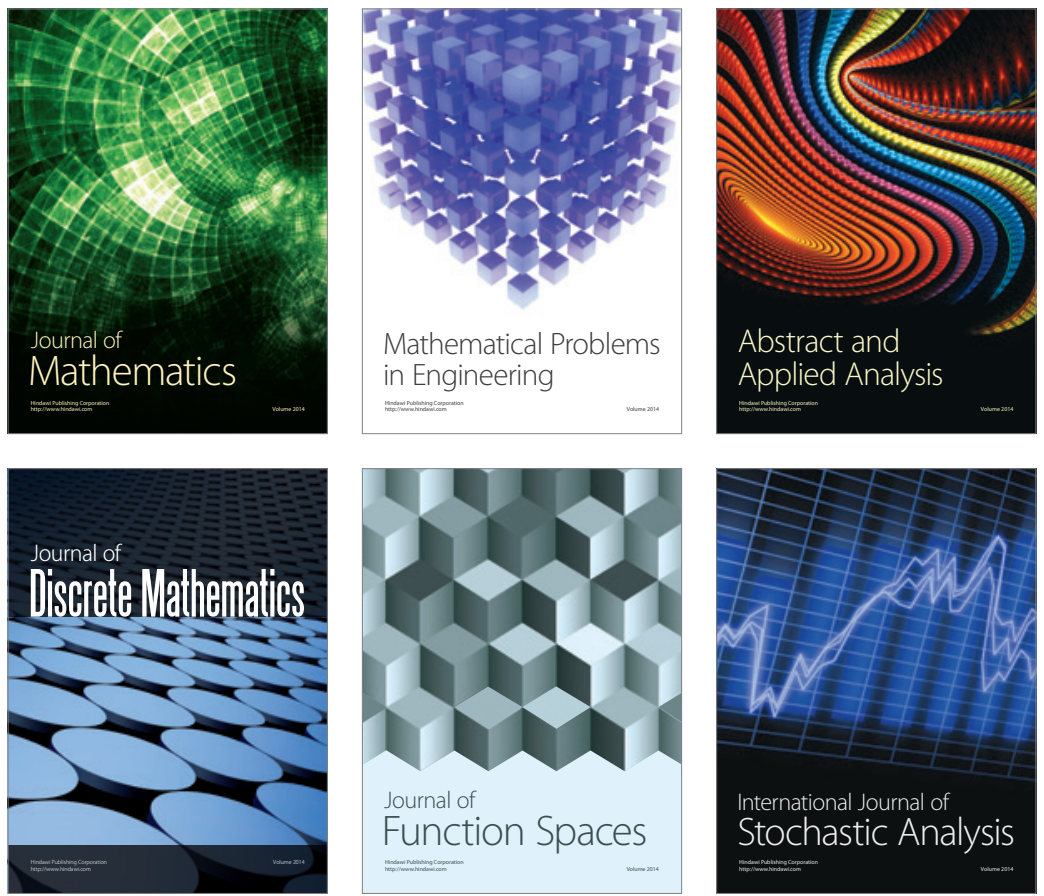

Journal of

Function Spaces

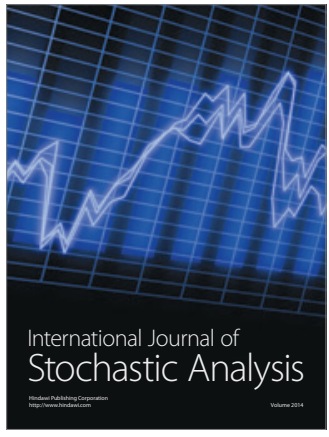

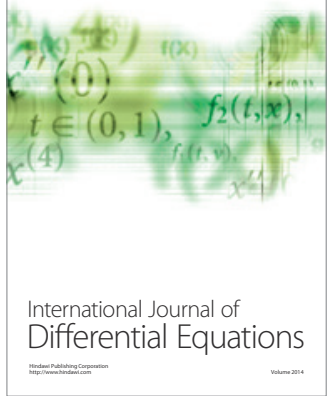
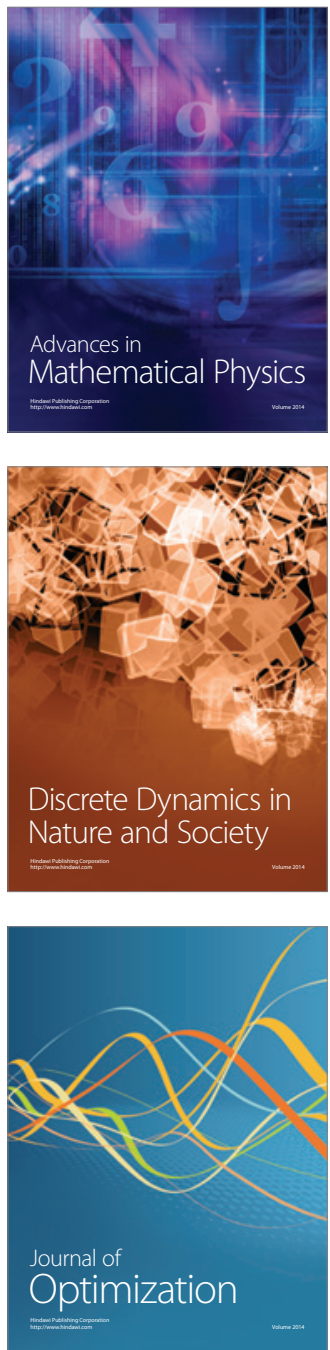